\title{
Proposed scaling law for intensity evolution in hadron storage rings based on dynamic aperture variation with time
}

\author{
M. Giovannozzi* \\ CERN, CH 1211 Geneva 23, Switzerland \\ (Received 22 March 2011; published 2 February 2012)
}

\begin{abstract}
A scaling law for the time dependence of the dynamic aperture, i.e., the region of phase space where stable motion occurs, has been proposed in previous papers [M. Giovannozzi, W. Scandale, and E. Todesco, Part. Accel. 56, 195 (1996); M. Giovannozzi, W. Scandale, and E. Todesco, in Proceedings of the 1997 Particle Accelerator Conference, edited by M. Comyn, M. K. Craddock, M. Reiser, and J. Thomson (IEEE Service Center, Piscataway, NJ, 1997), p. 1445; M. Giovannozzi, W. Scandale, and E. Todesco, Phys. Rev. E 57, 3432 (1998)]. This law, based on the analysis of numerical simulations data, is not entirely phenomenological, but motivated by some fundamental theorems of the theory of dynamical systems and indicates that the dynamic aperture has a logarithmic dependence on time. This result is used in turn as a basis for deriving a scaling law for the intensity evolution in hadron storage rings. This relationship is presented and discussed in detail in this paper. Furthermore, experimental data were compared to the predictions of this law and showed a remarkable agreement.
\end{abstract}

DOI: 10.1103/PhysRevSTAB.15.024001

\section{INTRODUCTION}

The prediction of particle stability and hence the evolution of the beam intensity in a synchrotron is still a challenge, both from the theoretical and the computational point of view. Many efforts have been devoted to this topic and a, certainly underestimated, sample of papers dealing with this topic can be found as Refs. [1-15], and references therein.

Different approaches have been applied, depending on whether the underlying dynamics is symplectic or not. In the latter case, e.g., for lepton machines where radiation damping cannot be neglected, it is customary to look for diffusion models explaining the drift of particles towards higher amplitudes [1-4,6,8,10,12-14]. These models arise naturally in all cases in which symplecticity is lacking. On the other hand, for symplectic systems, e.g., in the case of most hadron machines, radiation damping is negligible and hence a global diffusive model is not applicable. Of course, nonlinear effects, both external, e.g., due to the magnetic imperfections, or internal, such as space charge effects or beam-beam interactions, might act as sources of pseudodiffusive behavior, thus bringing particles towards higher amplitudes $[5,7,9,11,15]$. It is also clear that, due to the presence of invariants of the symplectic dynamics, it is in general excluded to have a global diffusive behavior. Rather, the increase of particles' amplitude occurs in small chaotic layers bounded by the invariants of the system.

\footnotetext{
*massimo.giovannozzi@cern.ch
}

Published by the American Physical Society under the terms of the Creative Commons Attribution 3.0 License. Further distribution of this work must maintain attribution to the author(s) and the published article's title, journal citation, and DOI.
PACS numbers: 29.20.db, 29.27.Bd, 05.45.-a, 45.05.+x

The nonlinearities of the beam dynamics give rise to the so-called dynamic aperture (DA), namely, the region in phase space where stable motion occurs. The concept of stable motion needs a proper definition of the time frame. In a mathematical sense, stable motion implies bounded motion for arbitrary time. In a more physical sense, particle stability can be linked to a maximum number of turns for which bounded motion occurs. Such a maximum number of turns would be defined on the basis of the specific application under consideration. Assuming this meaning for the rest of the paper, then the DA can be a function of time, with an asymptotic value representing the region of stability for any time.

It is clear that, whenever the dynamic aperture is inside the phase space region occupied by the beam, then particles will be pushed towards high amplitudes and hence lost, which leads to intensity reduction in a real machine.

The concept of DA has been used in many studies in order to minimize the effects of nonlinearities and hence to improve the machine performance, in theory and in practice. However, to the best of our knowledge, a direct quantitative relationship between intensity evolution and dynamic aperture is not yet available. It is worth stressing that such a relationship would be extremely useful, e.g., at the design study stage, where a link between dynamic aperture and beam lifetime would permit evaluation of the impact on performance of DA variation around the nominal design value.

In this paper we attempt to propose such a relationship. It is suggested that the time dependence of the beam intensity is related to the scaling law as proposed in the analysis of the time dependence of the dynamic aperture [16-18] obtained from numerical simulations. The key point is that, in spite of the simple form of such a scaling 
law, it is capable of reproducing many different types of behavior. Furthermore, a posteriori, a deeper justification in terms of two fundamental theorems of nonlinear beam dynamics was found. It must be emphasized here that the configuration considered throughout this paper is that dealing with a single beam of noninteracting particles under the influence of external nonlinear fields and for which the dynamic is symplectic. In particular, beam-beam interactions are neglected. It is also clear that the general problem addressed in this paper is very complex and the approach proposed here might not be suitable for all cases. Still, as will be shown, such an approach proves to be in rather good agreement with experimental data (see also Ref. [19], where preliminary results of this study were reported).

In Sec. II the scaling law for dynamic aperture is recalled and discussed. In Sec. III the proposed scaling law for the intensity as a function of time is presented assuming a Gaussian transverse distribution. Experimental data representing the time evolution of two real machines is analyzed using the proposed model and compared with the predictions of the standard diffusive models in Sec. IV. Finally, the conclusions are presented in Sec. V.

In Appendix A the Nekhoroshev theorem is presented with a discussion of some aspects relevant for this paper, while in Appendix B two models for the transverse beam distribution, different from the standard Gaussian, are considered and the corresponding scaling laws describing the intensity variation vs time are derived.

\section{TIME SCALING OF DYNAMIC APERTURE}

The starting point is the computation of the region in phase space where stable motion occurs. Different approaches were reviewed in Ref. [20] in order to define the most effective algorithm and also to associate a numerical error to the DA estimate. It is customary to assume a polar grid in normalized phase space:

$$
x=r \cos \theta \quad y=r \sin \theta \quad 0 \leq \theta \leq \pi / 2,
$$

where $x, y$ are expressed in units of $\sigma_{x}, \sigma_{y}$, respectively. Then, an ensemble of initial conditions defined on such a grid are tracked for up to $N_{\max }$ turns to assess their stability. If $r(\theta ; N)$ stands for the last stable amplitude for up to $N$ turns in the direction $\theta$, then the dynamic aperture can be defined as

$$
D(N)=\frac{2}{\pi} \int_{0}^{\pi / 2} r(\theta ; N) d \theta \equiv\langle r(\theta ; N)\rangle
$$

It is worth recalling that more refined algorithms can be devised, where the different directions in phase space have different weights.

According to the results of the studies reported in Refs. [16-18], the following scaling law holds:

$$
D(N)=D_{\infty}\left(1+\frac{b}{[\log N]^{\kappa}}\right),
$$

where $D_{\infty}$ represents the asymptotic value of the amplitude of the stability domain, while $b$ and $\kappa$ are additional parameters. These three parameters are obtained by fitting the results of numerical simulations. In Fig. 1 an example of the result of the DA computation and of the behavior of $D(N)$ is shown. The numerical simulations are performed on a model of the LHC machine including the measured magnetic errors for all classes of magnets. The optics configuration features three insertions, housing the ATLAS, ALICE, and CMS detectors, squeezed down to $\beta^{*}=2 \mathrm{~m}$, while the fourth one is at $\beta^{*}=10 \mathrm{~m}$, for the $\mathrm{LHCb}$ detector. The energy is $3.5 \mathrm{TeV}$, corresponding to the configuration for the 2010 LHC physics run (see Ref. [21] for more details on the configuration used). In the left-hand side of Fig. 1 the stable points in the $x-y$ space are reported. The red dots refer to the initial conditions that are stable up to $N_{\max }=10^{5}$, while the blue are unstable and their size has been chosen to be proportional to the actual stability duration time. Some scattered regions stable for only short times are clearly visible as is a welldefined, simply connected stable region. On the right-hand side the evolution of $D(N)$ is shown. The dots represent the numerically computed values of $D(N)$ according to Eq. (2), while the continuous line represents the fitted function based on Eq. (3), showing an excellent agreement with the numerical data. The dotted line represents $D_{\infty}$, the asymptote of the inverse logarithmic law. To be precise the fit provides

$$
D_{\infty}=23.74 \pm 0.01 \quad b=0.439 \pm 0.005 \quad \kappa=1.4 \pm 0.05 \text {, }
$$

where the errors on $D_{\infty}$ and $b$ are those associated to the least square fit. The exponent $\kappa$ is obtained by fixing it to an arbitrary value $\bar{\kappa}$, computing the best fit parameters $D_{\infty}(\bar{\kappa}), b(\bar{\kappa})$ and selecting the value of the exponent that provides the minimum residue of the fit. The associated error is not the standard error for a least square fit, but rather the step size in the scan performed over $\kappa$ in order to minimize the residues.

It is worth noting that the quantity $D(N)$ is invariant under transformation of the type $N \rightarrow N^{a}$, and $b \rightarrow a^{\kappa} b$.

The interesting point is that such a parametrization is compatible with the hypothesis that the phase space can be partitioned into two regions: a central core, with $r<D_{\infty}$, where Kolmogorov-Arnold-Moser (KAM) [22] surfaces confine the motion, thus producing a stable behavior apart from a set of small measure where Arnold diffusion can take place; an outer part, with $r>D_{\infty}$, where chaotic motion occurs and the escape rate to infinity is given by a Nekhoroshev-like estimate [23-25] such as

$$
N(r)=N_{0} \exp \left[\left(\frac{r_{*}}{r}\right)^{1 / \kappa}\right],
$$

where $N(r)$ is the number of turns that are estimated to be stable for particles with initial amplitude smaller than $r$. 

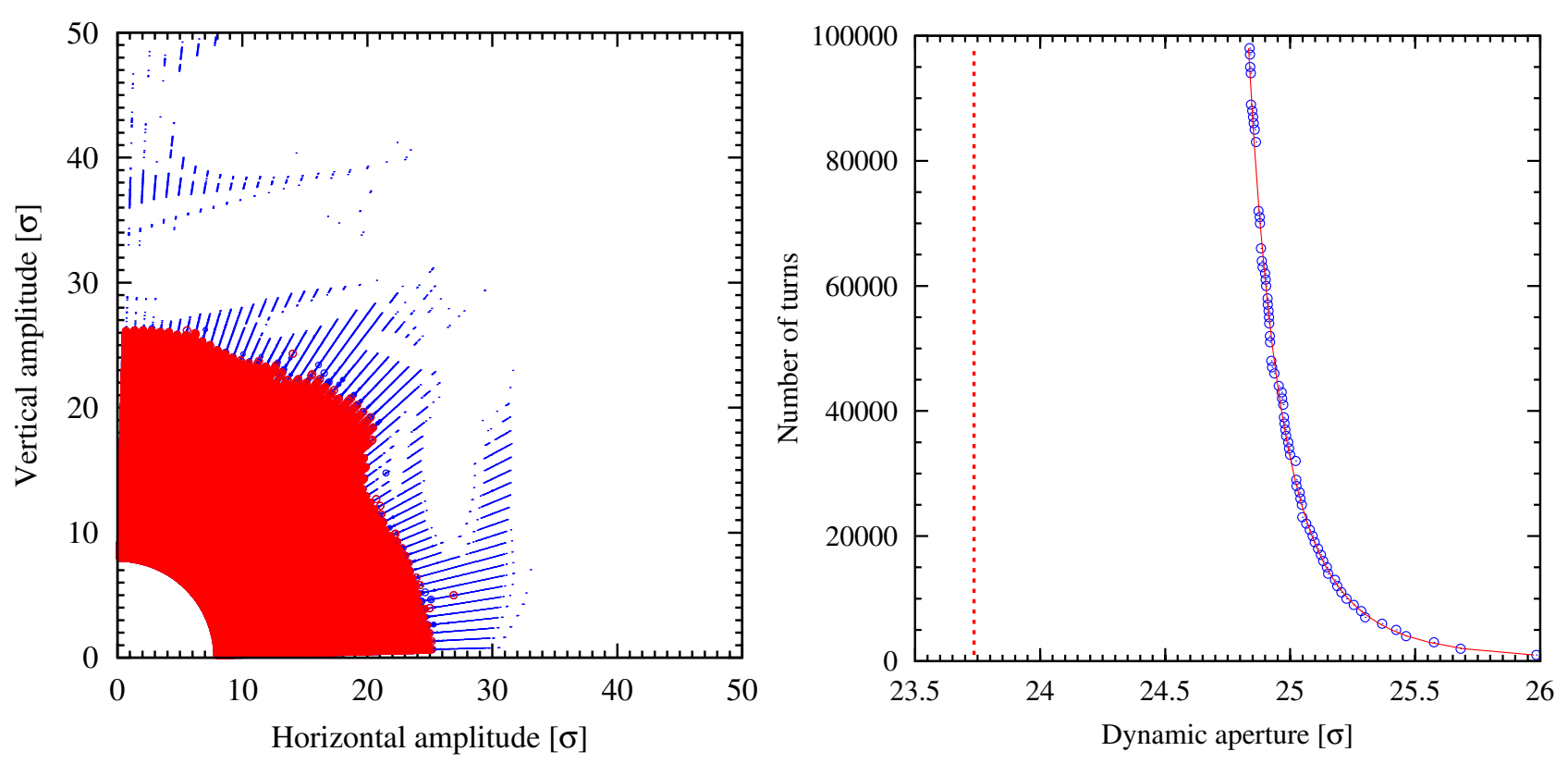

FIG. 1. Dynamic aperture of a model of the LHC ring (left) in physical space. The red points represent the initial conditions stable up to the maximum number of turns $\left(10^{5}\right)$ used in the numerical simulation. The blue points represent unstable conditions and their size is proportional to the number of turns by which their motion is still bounded. The time evolution of the DA is shown on the right. The markers represent the numerical results, while the continuous line shows the fitted inverse logarithmic law. The dotted line represents $D_{\infty}$.

This mathematical description can be translated into a more physical picture. Up to a certain amplitude, particle's motion in a storage ring is stable, i.e., bounded, at the level of the time scale given by the storage time, which is the only relevant time scale from a physical point of view. Beyond such a special amplitude, the nonlinear effects together with the associated resonances induce a pseudodiffusive motion. These phenomena induce a growth of the particle's amplitude and eventually lead to reaching the limiting apertures of the ring, thus producing beam losses.

The parameter $b$ in Eq. (3) is related to the width of the amplitude interval in which the pseudodiffusive behavior occurs. The speed of such a pseudodiffusion is given by the inverse logarithmic dependence on the number of turns and on the exponent $\kappa$. A large value of $\kappa$ implies a faster amplitude growth.

According to the Nekhoroshev-like estimate, the escape rate of particles located at small amplitude is exponentially long. Hence, to represent situations in which the size of the region stable for arbitrarily long number of turns shrinks to zero, it is necessary to assume that the parameters in the relationship (3) might undergo some drastic changes. For instance, instead of assuming that $D_{\infty}, b, \kappa$ are all positive, according to the proposed model based on KAM and Nekhoroshev theorems, some of them might become negative.

Alternatively, one could think of extending Eq. (3), well justified for positive values of the three parameters, also to negative values, thus going beyond what is the original justification. Even in this more comprehensive meaning of Eq. (3), not all possible combinations of signs of $D_{\infty}, b$, $\kappa$ provide meaningful behavior. Interestingly enough, two regimes were identified [18]: (i) in 4D systems the three quantities $D_{\infty}, b, \kappa$ are all positive [16,17] (this corresponds to having a stable region in phase space for an arbitrarily long time and hence being in the condition of applicability of the KAM and Nekhoroshev theorems); (ii) in $4 \mathrm{D}$ systems with tune modulation or $6 \mathrm{D}$ models, including off-momentum dynamics, it is possible that there is no stable region even for a finite number of turns [18]. This corresponds to one of the two cases:

$$
\begin{array}{lll}
D_{\infty}>0 & \kappa<0 & b<0 \\
D_{\infty} \leq 0 & \kappa>0 & b>0 .
\end{array}
$$

The first case represents a situation with global chaoticity [18]. In particular, the fact that tracking data could be fitted with negative $\kappa$ has been already observed several years ago (see discussion in Ref. [18]). The latter is compatible with a situation in which the stable KAM area shrinks to zero and the escape to infinity is governed by a Nekhoroshev-like behavior. Clearly, the possibility of having $D_{\infty}<0$ goes beyond the proposed picture based on phase space partitioning into KAM and Nekhoroshev regions.

\section{SCALING LAW OF BEAM INTENSITY AND LOSSES}

From the preceding discussion, it is possible to derive a model for the variation of the beam intensity due to the particle losses induced by the dynamic aperture. If the beam distribution is assumed to be Gaussian in $x$ and $y$, 


$$
\rho_{G}(x, y)=\frac{1}{2 \pi \sigma_{x} \sigma_{y}} e^{-\left[\left(x^{2} / 2 \sigma_{x}^{2}\right)+\left(y^{2} / 2 \sigma_{y}^{2}\right)\right]},
$$

then after transforming to polar coordinates and using the very definition of $D(N)$ (2), i.e., assuming that particles with amplitude beyond $D(N)$ at turn $N$ are lost, then the evolution of the beam intensity $I(N)$ can be found as

$$
\frac{I(N)}{I_{1}}=1-\int_{D(N)}^{+\infty} e^{-\left(r^{2} / 2\right)} r d r=1-e^{-D^{2}(N) / 2},
$$

where $I_{1}=I(1)$ and it is assumed that $D(N) \underset{N \rightarrow 1}{\longrightarrow}+\infty$ and $D(N)$ is assumed to be expressed in units of sigma.

\section{A. Positive dynamic aperture}

This case corresponds to the situation where all three parameters $D_{\infty}, b, \kappa$ are positive. Under this assumption, it is readily found that

$$
I_{\infty}=I_{1}\left(1-e^{-D_{\infty}^{2} / 2}\right) .
$$

In this scenario, some more insight can be gained by considering simply the expression for the relative losses at time $N$. From Eq. (8) it can be shown that

$$
\frac{\Delta I}{I_{1}}\left(N, D_{\infty}, b, \kappa\right)=e^{-D^{2}(N) / 2}
$$

and the total relative losses are given by

$$
\frac{\Delta I}{I_{1}}\left(\infty, D_{\infty}, b, \kappa\right)=e^{-\left(D_{\infty}^{2} / 2\right)} .
$$

The scaling law for the total relative losses can be found easily. In fact, if $D_{\infty} \rightarrow \alpha D_{\infty}$, which corresponds to assuming that the dynamic aperture is rescaled or changed due to a change in the dynamical system under consideration, then the losses will scale as

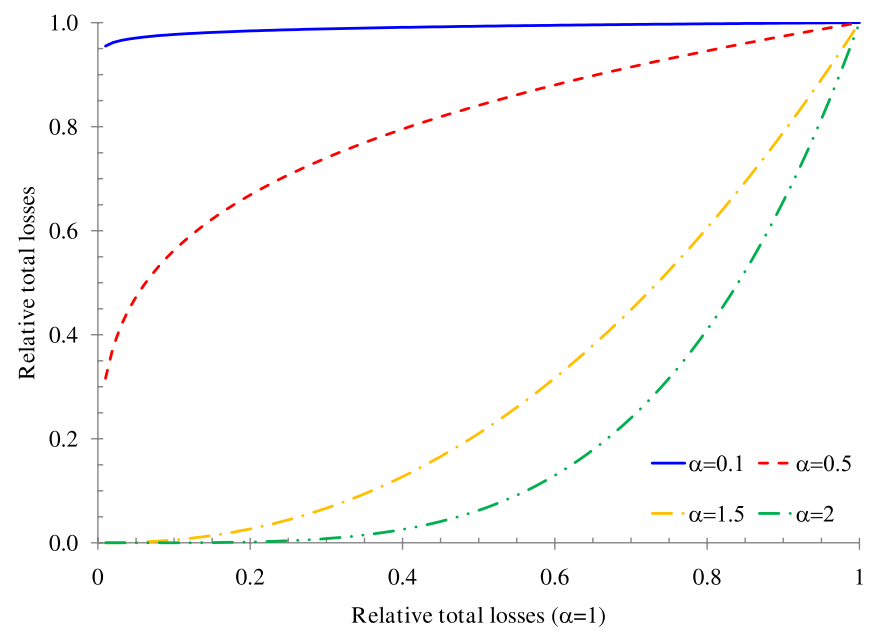

FIG. 2. Plot of $\frac{\Delta I}{I_{1}}\left(N, \alpha D_{\infty}, b, \kappa\right)$ vs $\frac{\Delta I}{I_{1}}\left(N, D_{\infty}, b, \kappa\right)$ for some values of $\alpha$ for a Gaussian distribution.

$$
\frac{\Delta I}{I_{1}}\left(N, \alpha D_{\infty}, b, \kappa\right)=\left[\frac{\Delta I}{I_{1}}\left(N, D_{\infty}, b, \kappa\right)\right]^{\alpha^{2}} .
$$

This equation shows that there is a strong dependence of the losses on the value of the dynamic aperture. In Fig. 2 the functions $\frac{\Delta I}{I_{1}}\left(N, \alpha D_{\infty}, b, \kappa\right)$ are plotted for four values of $\alpha$, illustrating a very strong dependence on this parameter.

It is also possible to linearize Eq. (12) around the nominal value of the dynamic aperture, corresponding to $\alpha=1$, obtaining

$$
\frac{\Delta I}{I_{1}} \approx\left(\frac{\Delta I}{I_{1}}\right)_{\alpha=1}+2\left(\frac{\Delta I}{I_{1}}\right)_{\alpha=1} \log \left(\frac{\Delta I}{I_{1}}\right)_{\alpha=1}(\alpha-1) .
$$

Of course, it could be argued that, in general, not only the dynamic aperture $D_{\infty}$ is affected by a change in the system's parameters, but also the constants in the logarithmic law, namely $b$ and $\kappa$. In this case, the scaling (12) is exact only for the total losses, as these depend only on the value of $D_{\infty}$. Indeed, if Eq. (10) is combined with (3), then no simple scaling can be found other than the following:

$$
\frac{\Delta I}{I_{1}}\left(N, D_{\infty}, b, \kappa\right) \rightarrow\left[I_{1} I_{2}^{\beta} I_{3}^{\beta^{2}}\right]^{\alpha^{2}}=\frac{\Delta I}{I_{1}}\left(N, \alpha D_{\infty}, \beta b, \kappa\right),
$$

with the following definitions:

$I_{1}=e^{-D_{\infty}^{2} / 2} \quad I_{2}=e^{-2 b D_{\infty}^{2} /\left(2 \log ^{\kappa} N\right)} \quad I_{3}=e^{-b^{2} D_{\infty}^{2} /\left(2 \log ^{2 \kappa} N\right)}$.

The invariance property quoted in the introduction implies that the general scaling holds:

$$
\frac{\Delta I}{I_{1}}\left(N^{\beta / \kappa}, \alpha D_{\infty}, \beta b, \kappa\right)=\left[\frac{\Delta I}{I_{1}}\left(N, D_{\infty}, b, \kappa\right)\right]^{\alpha^{2}},
$$

where the exponent $\kappa$ is always assumed constant as, from a theoretical point of view, it should be a function only of the number of degrees of freedom of the system under consideration. Hence, two systems with the same dimensionality can differ only by $D_{\infty}$ and $b$.

\section{B. Nonpositive dynamic aperture}

This case corresponds to the situation where not all of the three parameters $D_{\infty}, b, \kappa$ are positive. In this situation the relevant quantity is the time at which the dynamic aperture becomes zero, namely,

$$
D(\bar{N})=0 \quad \log \bar{N}=|b|^{1 / \kappa} .
$$

From the parametrization of the inverse logarithmic law, $\bar{N}$ is a function of only $b$ and $\kappa$, but not of $D_{\infty}$ which is of less importance in such a scenario. From Eq. (17) a scaling law for $\log \bar{N}$ can be derived and it reads

$$
\log \bar{N} \rightarrow|\beta|^{1 /(\gamma \kappa)} \log ^{1 / \gamma} \bar{N}
$$

if $b \rightarrow \beta b$ and $\kappa \rightarrow \gamma \kappa$. 
Similar computations can be carried out for other beam distributions, providing an analytical estimate for the intensity variation vs time as a function of the dynamic aperture. These results are given in Appendix B for the case of quasiparabolic [26,27] and Lévy-Student [28] distributions. The first can be considered a suitable model for representing beams collimated at a certain number of sigmas in the transverse dimensions. The latter is particularly suited to describing beams with heavy tails, i.e., tails more relevant than those of a Gaussian distribution, or halo (see, e.g., Ref. [29] for a discussion on halo definition).

\section{EXPERIMENTAL VERIFICATION}

The verification of the proposed scaling law for the time evolution of the beam intensity by means of experimental data is not easy. In particular, the law may not apply to the existing data sets as the experimental conditions might not fit the necessary assumptions, such as the absence of nonlinear effects other than magnetic nonlinearities, and beam intensity low enough to exclude the presence of collective effects. Furthermore, the few data sets that were found in the literature do not report the details of the transverse beam distribution. For this reason, the fit of the scaling law of the intensity vs time to only a Gaussian transverse beam distribution will be attempted.

\section{A. Fermilab Tevatron data}

After a search in the literature, an interesting data set was found in Ref. [30]. There, intensity vs time for the Tevatron machine with the proton beam only at injection energy was reported. These measurements fulfill the conditions necessary for the application of the approach proposed in this paper. In fact, the only sources of beam losses are the nonlinear effects due to the magnetic field quality, and are therefore related to dynamic aperture. No beambeam related effect is relevant. Furthermore, the interesting point is that in the paper [30] the intensity evolution was analyzed using a diffusive model with properties derived by matching the model to the experimental observations. Three functions have been proposed in Refs. [30,31], namely: (i) pure exponential, $I(t) \propto e^{-t / \tau}$ (this corresponds to a single component of the infinite series representing the solution of a diffusion equation with a constant diffusion coefficient representing the beam motion close to the dynamic aperture); (ii) square root function, $I(t) \propto$ $(1-\sqrt{t / \tau})$ (this is shown to be a good approximation of the solution of the diffusion equation [31]); (iii) exponential with square root time dependence, $I(t) \propto$ $e^{\sqrt{t / \tau}}$ (this is shown to be a good approximation of the series, truncated to a rather high order, representing the general solution of the diffusion equation).

The agreement between experimental data and the proposed diffusive model is very good for the last two functions presented before.
Of course, as the currently proposed approach assumes a pseudodiffusive behavior $\grave{a}$ la Nekhoroshev, it is natural to believe that a good agreement would be found by the new approach. Equation (8) is used to fit the data by adjusting the free parameters $D_{\infty}, b$, and $\kappa$. Indeed, in order to improve the numerical convergence of the nonlinear fit, the parameters $D_{\infty}, b$ were computed for fixed $\kappa$, which is then varied and its final value obtained by minimizing the fit residuals.

The best result gives a residue of $4.6 \times 10^{-7}$. This should be compared with the residuals for the functions proposed in Ref. [30] that are of the order of 5.5-6.3 $\times$ $10^{-7}$. This indicates that the proposed approach is at least as good as the standard diffusive models.

In Fig. 3 the experimental data, the fit functions proposed in Ref. [30], and that based on the inverse logarithmic law are shown. The agreement is remarkable for all models, but in particular for the new proposal of this paper.

It is worth noting that the asymptotic dynamic aperture is positive, thus indicating that the motion is globally stable. Furthermore, the large value of $b$ indicates that stochastic motion is occurring in a rather wide region of phase space. Also, the value of $\kappa$ agrees well with the theoretical estimate given by the number of degrees of freedom of the system under consideration.

One might argue the residue depends critically on the number of free parameters of the time-evolution law, which is three for the approach based on the inverse logarithmic scaling of DA, and only two for the case of the diffusive models proposed in Ref. [30]. However, it was tested that the numerical dependence of the residue on $\kappa$ is indeed rather weak: a variation of a factor of 2 around the optimal $\kappa$ induces a change in the residue of only $10 \%-20 \%$. This indicates that the logarithmic time dependence is the key of the good agreement and the exponent $\kappa$ provides only a refinement.

A summary of the fit parameters is reported in Table I.

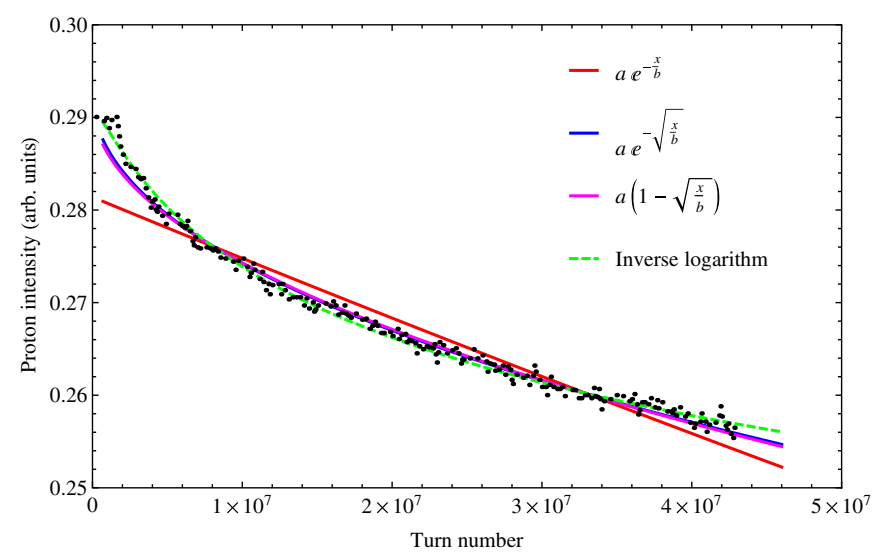

FIG. 3. Beam intensity evolution for the Tevatron at injection energy from Ref. [30] (black dots) together with the interpolated functions proposed therein and the one proposed in this paper. The agreement is remarkable. 
TABLE I. Fit parameters for the functions used to model the Tevatron data (IL stands for the inverse logarithmic scaling law proposed in this paper).

\begin{tabular}{lcccc}
\hline \hline Relation & $a / D_{\infty}$ & $b$ & $\kappa$ & $10^{-7}$ \\
\hline$a e^{-(t / b)}$ & $0.2814 \pm 3 \times 10^{-4}$ & $42.1 \pm 0.7$ & & 38 \\
$a e^{-\sqrt{t / b}}$ & $0.2925 \pm 2 \times 10^{-4}$ & $239 \pm 3$ & & 7.3 \\
$a(1-\sqrt{t / b})$ & $0.2916 \pm 2 \times 10^{-4}$ & $283 \pm 4$ & & 8.6 \\
$\mathrm{IL}-$ Gaussian & $1.10 \pm 0.01$ & $645 \pm 7$ & $3.24 \pm 0.01$ & 5.4 \\
\hline \hline
\end{tabular}

\section{B. CERN SPS data}

A second set of experimental data was taken from beam measurements performed at the CERN Super Proton Synchrotron (SPS). Recently, a number of tests were made in view of assessing whether the beam conditions at the SPS are suitable for a realistic test of crab cavities [32]. The idea is to use the SPS as LHC test bed and verify the impact of crab cavities on the dynamics of a proton beam. During these tests, a continuous loss of beam intensity for a single-bunch beam during a coast at $55 \mathrm{GeV}$ was reported [33]. These data [34] have been considered a useful benchmark for the approach presented here. In Fig. 4 the experimental data (markers) are shown together with predictions from diffusive models and the inverse logarithmic variation of DA. The first interesting feature is that the second derivative with respect to time of intensity change is negative, while it was positive for the data from the Tevatron. This characteristic rules out immediately the diffusive models proposed in Ref. [30]. In fact, they impose a positive second order time derivative of intensity and this is clearly seen in Fig. 4. On the other hand, the proposed approach is capable of fitting the measured data very accurately. Interestingly enough, both $b$

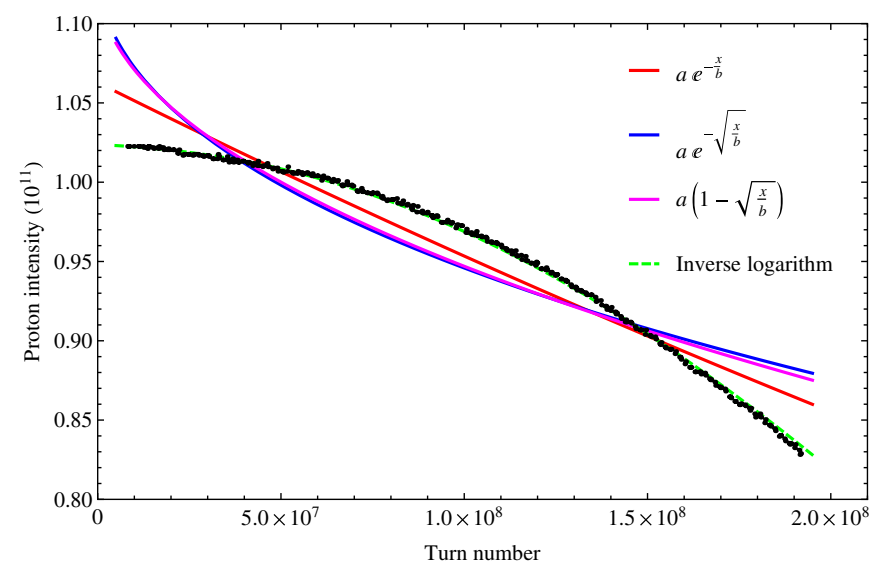

FIG. 4. Beam intensity evolution for the CERN SPS at $55 \mathrm{GeV}$ (black dots) during a coast with a single bunch. The same interpolation functions used for the Tevatron data are used here. Only the proposed model based on the inverse logarithmic scaling law for dynamic aperture is in good agreement with the experimental data. and $\kappa$ are negative, thus indicating that no stable region exists for the SPS. Based on the experience with the analysis of tracking data, in which negative values of $\kappa$ and $b$ are found whenever modulational effects are present (e.g., transverse tune modulation due to either ripple on quadrupoles or coupling to longitudinal motion via chromaticity [18]), one would conclude that the observed SPS behavior is probably linked to some time-dependent effect such as tune modulation.

Applying Eq. (17) it is possible to estimate the time for which $D(N)=0$ and the intensity will be equal to zero. This corresponds to $\approx 7.7 \times 10^{8}$ turns or equivalently $\approx 4.9$ hours of storage time.

Following the initial tests at $55 \mathrm{GeV}$, new measurements at higher energy were attempted with the goal of finding a better situation in terms of beam losses and beam lifetime. Long coasts at $270 \mathrm{GeV}$ were made with single-bunch proton beams and the data analyzed using the approach presented in this paper. The results are presented in Fig. 5. The experimental data are shown together with the results of the fit functions based on diffusive models and the inverse logarithm scaling law. In this case the intensity reduction is much smaller than for the $55 \mathrm{GeV}$ measurements and also the second order derivative is very much reduced. This, in turns, makes it possible to have a good agreement between the fitted functions based on the diffusive models and the data. On the other hand, the proposed fit is providing very good agreement, too. Applying Eq. (17) it is possible to estimate also in this case the time for which $D(N)=0$. This corresponds to $\approx 2.5 \times 10^{11}$ turns or equivalently $\approx 1.6 \times 10^{3}$ hours of storage time. Clearly, the losses at higher energy are much smaller than at $55 \mathrm{GeV}$ and the overall beam stability is greatly enhanced. It is worth emphasizing that the proposed explanation, based on external tune ripple, for the strong losses and lifetime limitation of the beam at $55 \mathrm{GeV}$ could also

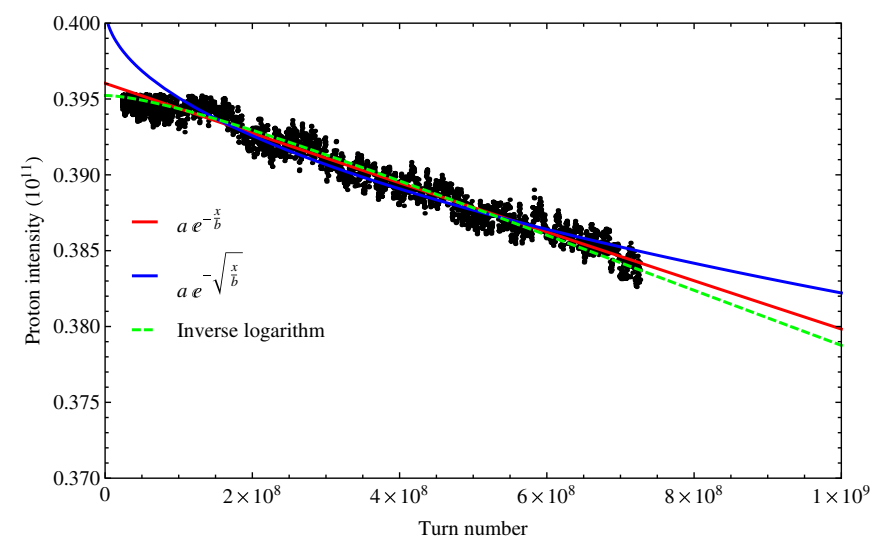

FIG. 5. Beam intensity evolution for the CERN SPS at $270 \mathrm{GeV}$ (black dots) during a coast with a single bunch. The good agreement between the measured intensity evolution and the proposed model based on the inverse logarithmic scaling law for dynamic aperture is clearly visible. 
TABLE II. Fit parameters for the functions used to model the CERN SPS data (IL stands for the inverse logarithmic scaling law proposed in this paper).

\begin{tabular}{lcccr}
\hline \hline Relation & & & Fit parameters & \\
Energy [GeV] & $a / D \infty$ & $b$ & $\begin{array}{c}\text { Residue } \\
10^{-7}\end{array}$ \\
\hline $\mathrm{IL}$-Gaussian & 55 & $4.185 \pm 0.006$ & $(-1.660 \pm 0.006) \times 10^{-8}$ & $-8.2 \pm 0.1$ \\
$a e^{-(t / b)}$ & 270 & $0.39604 \pm 3 \times 10^{-5}$ & $2393 \pm 11$ & 34 \\
$a e^{-\sqrt{t / b}}$ & 270 & $0.40117 \pm 6 \times 10^{-5}$ & $42680 \pm 462$ & 5.2 \\
$\mathrm{IL}$-Gaussian & 270 & $8.96 \pm 0.05$ & $(-331 \pm 2) \times 10^{-4}$ & $-1.4 \pm 0.1$ \\
\hline \hline
\end{tabular}

explain why the harmful effect would decrease with energy. In fact, the accuracy of the power converters improves at high energy and the beam becomes more rigid and less sensitive to external perturbations.

The fitted parameters are listed in Table II. It is interesting to note that the values of $D_{\infty}$ for the 55 and $270 \mathrm{GeV}$ cases scale almost perfectly as $\sqrt{\beta \gamma}$, which could be interpreted as a sign that the machine intrinsic behavior, i.e., its dynamic aperture in millimeters, is constant, while the external perturbation, i.e., the tune ripple, is improving, thus making the intensity loss better at high energy.

\section{CONCLUSIONS}

In this paper an attempt to establish a link between the value of the dynamic aperture and the beam losses has been presented. Rather than using a diffusive model to describe the particle's motion and, hence, derive the evolution of the beam intensity, the inverse logarithmic decay of the dynamic aperture as a function of turn number is used.

A relationship between the relative losses (total or up to turn number $N$ ) and the dynamic aperture is obtained so that a scaling law can be derived for the case of positive dynamic aperture. According to such a scaling law, the dependence on the dynamic aperture is rather strong and its variation around the nominal value induces a large relative variation of the expected beam losses.

For the case of nonpositive dynamic aperture, a scaling law of the logarithm of the turn number $\bar{N}$ at which $D(\bar{N})=0$ is derived. The proposed scaling law can be generalized to non-Gaussian transverse beam distributions, namely, quasiparabolic, simulating beams with truncated tails, and Lévy-Student, simulating beam with heavy tails.

The proposed inverse logarithmic dependence of intensity vs time has been applied to two experimental data sets, one from Tevatron at injection energy and one from SPS during coasts at 55 and $270 \mathrm{GeV}$. In spite of the differences between the two types of time evolution of the beam intensity, the proposed model fits the experimental observations rather well. For the sake of completeness, in the case of the Tevatron data a similar good agreement is found with predictions based on diffusive models. The diffusive models cannot explain the experimental observations for the SPS at $55 \mathrm{GeV}$, while a good agreement could be found at $270 \mathrm{GeV}$. In both cases, however, the proposed fit with the inverse logarithm law provides a very good agreement with the measured data. The fitted parameters clearly show an improvement of the beam stability, and hence beam losses, at higher energy.

It is worthwhile emphasizing that the proposed model for the evolution of the beam intensity is not entirely phenomenological, but motivated by two fundamental and very general theorems of nonlinear dynamics and it could be made even more general.

Such a mathematical picture can be turned into a physical description of the underlying processes. The interplay of the nonlinear effects and the resonances generate a growth of the particle's amplitude that is similar to a diffusive process. However, such a growth occurs only beyond a certain threshold amplitude, below which no growth is generated and no particle loss observed. Hence, in case the threshold amplitude happens to be located in the region occupied by the beam, then losses are produced.

The direct relationship between intensity variation with time and DA could be used to perform measurements of the dynamic aperture in a hadron machine by fitting intensity curves over a sufficiently long interval of time.

\section{ACKNOWLEDGMENTS}

Fruitful discussions with S. Fartoukh, W. Scandale, E. Todesco, and F. Zimmermann are warmly acknowledged. We give a special thanks to V. Shiltsev for comments on the original manuscript. I would like to express my gratitude to E. McIntosh for carefully reading the manuscript and for the many comments and improvements. I am also particularly indebted to E. Métral for providing me with the data taken at the CERN SPS and presented in this paper. Finally, I would like to express my warmest thanks to the two anonymous referees who helped improve the manuscript with stimulating comments.

\section{APPENDIX A: SOME COMMENTS ON THE NEKHOROSHEV THEOREM}

In this Appendix the statement of the Nekhoroshev theorem [23] is reported and commented. The key result is expressed as follows.

Theorem 1.-Given a Hamiltonian in the form of $H_{0}(J)+\epsilon H_{1}(J, \phi)$, where $J$ and $\phi$ represent action angle variables and $H_{0}$ stands for the integrable part of the 
Hamiltonian. Provided that $H_{0}$ satisfies certain geometrical conditions, also called steepness conditions [23], then there are positive constants $a, b, \epsilon_{0}$, such that if $0<\epsilon<\epsilon_{0}$ the solutions $J(t), \phi(t)$ satisfy

$$
|J(t)-J(0)|<\epsilon^{b}
$$

for all $t \in[0, T]$, where

$$
T=\frac{1}{\epsilon} \exp \left(\frac{1}{\epsilon^{a}}\right) .
$$

The two constants $a, b$ depend only on $H_{0}$ and can be expressed as

$$
a=\frac{2}{12 \zeta+3 s+14}, \quad b=\frac{3 a}{2 \alpha_{s-1}},
$$

where $s$ stands for the number of degrees of freedom and

$$
\begin{aligned}
\zeta= & \left(\alpha_{1}\left\{\alpha_{2} \cdots\left[\alpha_{s-3}\left(\alpha_{s-2} s+s-2\right)+s-3\right]+\cdots+2\right\}+1\right) \\
& -1 .
\end{aligned}
$$

The quantities $\alpha_{j}$ are related to the steepness conditions, thus indicating that the estimates (A1) and (A2) are closely linked with the geometrical properties of the function $H_{0}$. The conditions (A1) and (A2) mean that it is possible to provide an upper bound to the variation of the action of the system for an exponentially long interval of time.

It is also worth emphasizing that the two constants $a$ and $b$ are not independent and that $a$ decreases when increasing the number of degrees of freedom such that the estimate (A2) becomes worse as the number of degrees of freedom increases. In Ref. [23] it is also mentioned that sharper estimates like

$$
a=\frac{1}{3 \zeta+s+4}-\sigma
$$

with $\sigma$ a positive, but arbitrarily small quantity, could be obtained, but no proof is given. This indicates that various expressions for the key parameters $a$ and $b$ can be obtained, but it is not obvious to prove mathematically that any of these forms of the parameters $a$ and $b$ is optimal, in the sense that no sharper value can be found.

It is also clear that whenever $a$ would become negative the same would happen for $b$, and hence the parameter $T$ would not be exponentially large anymore. Moreover, the bound to the action variation (A1) effectively would not be a bound anymore, as $\epsilon^{-b}$ would be an arbitrarily large quantity. Therefore, under the condition that $a$ is negative the whole idea behind the Nekhoroshev theorem would break down and no limit to action variation can be given. This is indeed compatible with the picture of global diffusion proposed in the paper for negative exponents.

It is worth mentioning that in Refs. [24,25] a different approach was used, as, instead of flows, maps are used and the Nekhoroshev theorem [23] is proved for time-discrete systems. The key point is that a more direct link between the exponent $a$ and the phase space dimensionality is established (see also Ref. [35]). In particular, it can be shown that $[24,25]$

$$
a=\frac{2}{1+\eta},
$$

where $\eta \leq s$ and $\eta$ is linked to the number-theoretical properties of the frequencies characterizing $H_{0}$. Also in this case, no unique estimate for $a$ is given, but rather a lower bound based on the system dimensionality. Therefore, as the link between the parameter $\kappa$ used in this paper and $a$ is $\kappa=1 / a$, one can derive an estimate for $\kappa$ given by

$$
\kappa \approx \frac{s+1}{2} .
$$

\section{APPENDIX B: THE CASE OF OTHER BEAM DISTRIBUTIONS}

The case of a Gaussian distribution is the standard and certainly the most appropriate model for describing the transverse beam distribution. However, in a number of situations the tails might feature a special behavior. Then, the so-called quasiparabolic $[26,27]$ or the generalized Lévy-Student [28] distributions will be best suited to modeling the actual transverse beam distribution. It is worth stressing that neither of the special classes of beam distributions are independent, meaning that they cannot be expressed as the product of two functions each depending on $x$ and $y$ only.

\section{Quasiparabolic}

The first model is suited to deal with truncated tails, satisfying also the additional constraint of providing a smooth distribution function at the location where it becomes zero. The explicit form in standard coordinates is

$\rho_{Q P, n}(x, y)=\frac{1}{2 \pi \sigma_{x} \sigma_{y}}\left(\frac{n+1}{n}\right)\left[1-\frac{1}{n}\left(\frac{x^{2}}{2 \sigma_{x}^{2}}+\frac{y^{2}}{2 \sigma_{y}^{2}}\right)\right]^{n}$

with the additional condition that

$$
\frac{x^{2}}{2 \sigma_{x}^{2}}+\frac{y^{2}}{2 \sigma_{y}^{2}} \leq n .
$$

This form of the quasiparabolic distribution is suggested by imposing that $\rho_{Q P, n}(x, y) \underset{n \rightarrow+\infty}{\longrightarrow} \rho_{G}(x, y)$, hence recovering the standard Gaussian distribution in Eq. (7). It is also easy to verify that the following holds:

$$
\sigma_{Q P, n, x / y}=\sqrt{\frac{n}{n+2}} \sigma_{x / y}
$$

connecting the sigma of the quasiparabolic distribution to the one of the standard Gaussian $\sigma_{x / y}$, and in the limit for $n \rightarrow+\infty, \sigma_{Q P, n}$ tends to the corresponding value for the 
Gaussian distribution. However, for each finite value $n$ the two are different. It is then possible to reexpress Eq. (B1) in terms of $\sigma_{Q P, n}$, giving

$$
\begin{aligned}
\rho_{Q P, n}(x, y)= & \frac{1}{2 \pi \sigma_{Q P, n, x} \sigma_{Q P, n, y}}\left[\frac{(n+1)(n+2)}{n^{2}}\right] \\
& \times\left[1-\frac{1}{n+2}\left(\frac{x^{2}}{2 \sigma_{Q P, n, x}^{2}}+\frac{y^{2}}{2 \sigma_{Q P, n, y}^{2}}\right)\right]^{n} .
\end{aligned}
$$

It is also worth emphasizing that the condition on the validity of $\rho_{Q P, n}(x, y)$ could also be expressed using the unit step function [defined as $\theta(x)=1, \forall x \geq 1$ and 0 in all other cases], hence multiplying the expression (B1) by $\theta\left\{n+2-\left[x^{2} /\left(2 \sigma_{Q P, n, x}^{2}\right)\right]+\left[y^{2} /\left(2 \sigma_{Q P, n, y}^{2}\right)\right]\right\}$.

Equation (8) can be recast in the following form:

$$
\frac{I(N)}{I_{1}}=1-\left[1-D^{2}(N)\left(\frac{n+2}{2 n^{2}}\right)\right]^{n+1},
$$

where a transformation to normalized (with respect to initial sigma) and then polar coordinates is performed. It is worth pointing out that expression (B5) tends to (8) in the limit of large $n$.

Unlike the case of Gaussian transverse beam distribution, $I_{1}$ represents the intensity corresponding to the number of turns $\bar{N}$ satisfying the condition $D(\bar{N})=\sqrt{2 n}$. In fact, for shorter times the dynamic aperture is larger than the extension of the beam distribution and hence no reduction in intensity is possible. For this new model, the simple scaling law for total relative losses (12) does not hold anymore and it is replaced by the more complicated expression

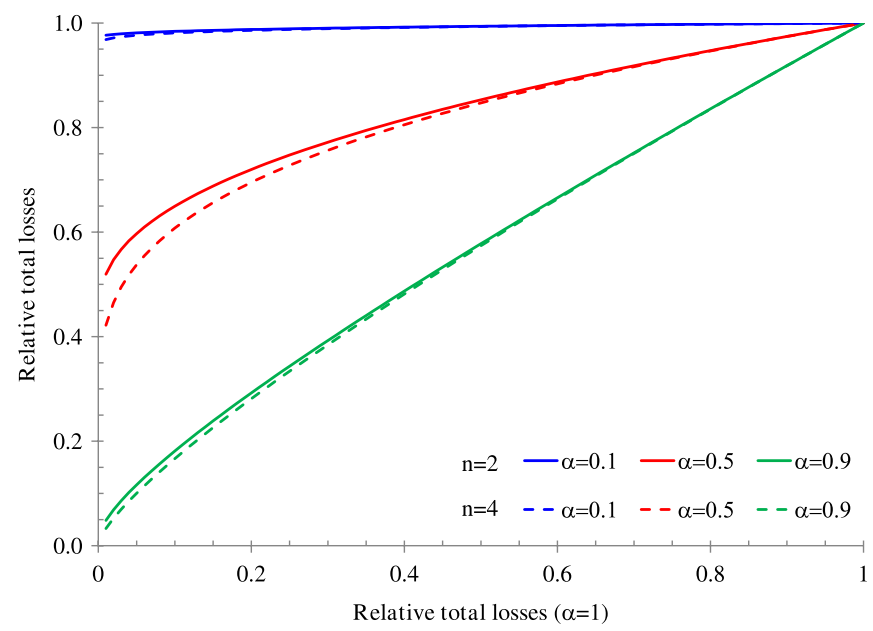

FIG. 6. Plot of $\frac{\Delta I}{I_{1}}\left(N, \alpha D_{\infty}, b, \kappa\right)$ vs $\frac{\Delta I}{I_{1}}\left(N, D_{\infty}, b, \kappa\right)$ for some values of $\alpha$ and $n$ for quasiparabolic distributions.

$$
\begin{aligned}
& \frac{\Delta I}{I_{1}}\left(N, \alpha D_{\infty}, b, \kappa\right) \\
& \quad=\left(1-\alpha^{2}\right)^{n+1}\left[1+\frac{\alpha^{2}}{1-\alpha^{2}} \frac{\Delta I}{I_{1}}\left(N, D_{\infty}, b, \kappa\right)^{1 /(n+1)}\right]^{n+1}
\end{aligned}
$$

for the case of positive dynamic aperture. In Fig. 6 the evolution of the total losses for different values of the parameter $\alpha$ is shown for two cases of order $n$. A weak dependence on $n$ is found, while $\alpha$ is still a crucial parameter, even if less so than for the Gaussian case.

Finally, it is worth stressing that the condition $D_{\infty}<$ $\sqrt{2 n}$ must hold true, otherwise Eq. (B5) is not valid. Furthermore, $\alpha<\sqrt{2 n} / D_{\infty}$ so that Eq. (B6) is also meaningful.

\section{Lévy-Student}

The presence of halo in the beam distribution is normally linked to the effect of space charge or other collective effects, such as beam-beam. In this case the tails are heavier than for a Gaussian model and it was proposed in Ref. [28] to use generalized Lévy-Student distributions. In this paper the following form is used:

$$
\rho_{L S, \nu, a}(x, y)=\frac{\sigma^{2}}{2 \pi \sigma_{x} \sigma_{y}} \frac{\nu a^{\nu}}{\left[a^{2}+x^{2}\left(\frac{\sigma}{\sigma_{x}}\right)^{2}+y^{2}\left(\frac{\sigma}{\sigma_{y}}\right)^{2}\right]^{(\nu+2) / 2}},
$$

where $\nu$ is a real quantity greater than 2 in order to have a finite sigma and under these conditions $a^{2}=(\nu-2) \sigma^{2}$. It is easy to verify that this form implies that the following limit holds $\rho_{L S, \nu, a}(x, y) \underset{\nu \rightarrow+\infty}{\longrightarrow} \rho_{G}(x, y)$. Furthermore, it can be found that

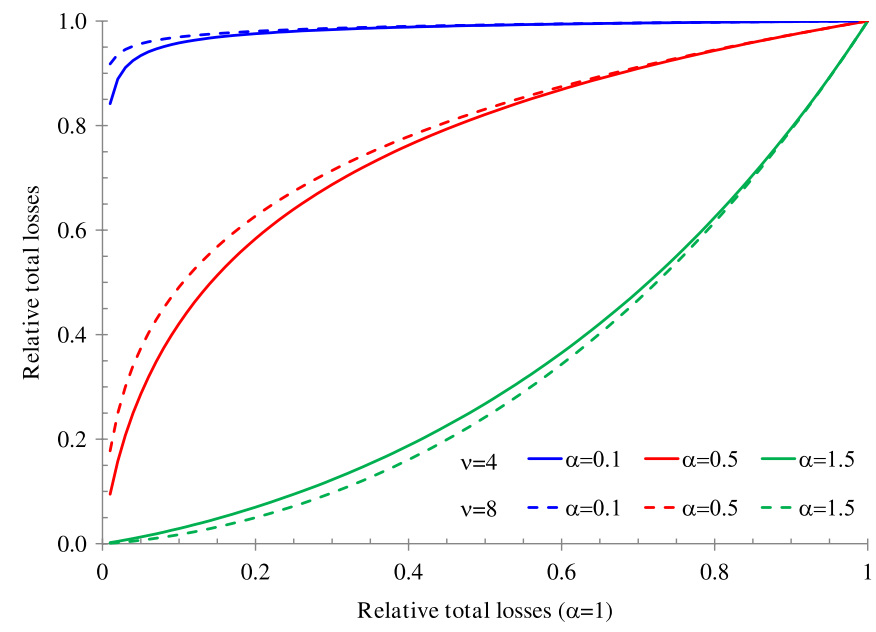

FIG. 7. Plot of $\frac{\Delta I}{I_{1}}\left(N, \alpha D_{\infty}, b, \kappa\right)$ vs $\frac{\Delta I}{I_{1}}\left(N, D_{\infty}, b, \kappa\right)$ for some values of $\alpha$ and $\nu$ for Lévy-Student distributions. 


$$
\sigma_{L S, \nu, a, x / y}=\sigma_{x / y}
$$

independently on the value of $\nu$ and $a$.

Finally Eq. (8) can be recast in the following form:

$$
\frac{I(N)}{I_{1}}=1-\frac{1}{\left[1+\frac{D^{2}(N)}{a^{2}}\right]^{\nu / 2}},
$$

where the customary transformation to normalized and then polar coordinates are applied.

In this case the tails extend to infinity as for the Gaussian case and hence $I_{1}=I(1)$. Also for this model the simple scaling law for total relative losses (12) does not hold anymore and it is replaced by

$$
\begin{aligned}
& \frac{\Delta I}{I_{1}}\left(N, \alpha D_{\infty}, b, \kappa\right) \\
& \quad=\frac{1}{\left(1-\alpha^{2}\right)^{\nu / 2}} \frac{1}{\left[1+\frac{\alpha^{2}}{1-\alpha^{2}} \frac{1}{\left(\Delta I / I_{1}\right)\left(N, D_{\infty}, b, \kappa\right)^{2 / \nu}}\right]^{\nu / 2}}
\end{aligned}
$$

for the case of positive dynamic aperture.

In Fig. 7 the functions in Eq. (B10) are shown. The dependence on $\nu$ is rather mild, while the curves for the values of $\alpha$ resemble very much those of the corresponding Gaussian case shown in Fig. 2.

[1] K. Hirata, in Proceedings of the Joint U.S.-CERN-JapanRussia School on Particle Accelerators, edited by S.I. Kurokawa, S. Y. Lee, E. Perevedentsev, and S. Turner (World Scientific, New Jersey, 1999).

[2] A. L. Gerasimov and N.S. Dikansky, Nucl. Instrum. Methods Phys. Res., Sect. A 292, 209 (1990).

[3] A. L. Gerasimov and N.S. Dikansky, Nucl. Instrum. Methods Phys. Res., Sect. A 292, 221 (1990).

[4] A. L. Gerasimov and N.S. Dikansky, Nucl. Instrum. Methods Phys. Res., Sect. A 292, 233 (1990).

[5] Y. Papaphilippou and F. Zimmermann, Phys. Rev. ST Accel. Beams 2, 104001 (1999).

[6] J. Gao, Nucl. Instrum. Methods Phys. Res., Sect. A 463, 50 (2001).

[7] Y. Papaphilippou and F. Zimmermann, Phys. Rev. ST Accel. Beams 5, 074001 (2002).

[8] C. Steier, D. Robin, L. Nadolski, W. Decking, Y. Wu, and J. Laskar, Phys. Rev. E 65, 056506 (2002).

[9] R. Assmann, F. Schmidt, and F. Zimmermann, in Proceedings of Eighth European Particle Accelerator Conference, edited by C. Petit-Jean-Genaz (Institute of Physics, London, UK, 2002), p. 1326.

[10] A. W. Chao, Phys. Rev. ST Accel. Beams 6, 094001 (2003).

[11] T. Sen, B. Erdelyi, M. Xiao, and V. Boocha, Phys. Rev. ST Accel. Beams 7, 041001 (2004).

[12] T.-Y. Lee, Phys. Rev. E 69, 046501 (2004).
[13] J. Gao, Nucl. Instrum. Methods Phys. Res., Sect. A 533, 270 (2004).

[14] T.-Y. Lee, J. Choi, and H. S. Kang, Nucl. Instrum. Methods Phys. Res., Sect. A 554, 85 (2005).

[15] W. Fischer, J. Beebe-Wang, Y. Luo, S. Nemesure, and L. Rajulapati, in Proceedings of the First International Particle Accelerator Conference, edited by A. Noda, C. Petit-Jean-Genaz, V. Schaa, T. Shirai, and A. Shirakawa (Institute of Physics, London, UK, 2010), p. 4752.

[16] M. Giovannozzi, W. Scandale, and E. Todesco, Part. Accel. 56, 195 (1996)

[17] M. Giovannozzi, W. Scandale, and E. Todesco, in Proceedings of the 1997 Particle Accelerator Conference, edited by M. Comyn, M. K. Craddock, M. Reiser, and J. Thomson (IEEE Service Center, Piscataway, NJ, 1997), p. 1445.

[18] M. Giovannozzi, W. Scandale, and E. Todesco, Phys. Rev. E 57, 3432 (1998).

[19] M. Giovannozzi, in Proceedings of the First International Particle Accelerator Conference (Ref. [15]), p. 4704.

[20] E. Todesco and M. Giovannozzi, Phys. Rev. E 53, 4067 (1996).

[21] M. Giovannozzi, in Proceedings of the First International Particle Accelerator Conference (Ref. [15]), p. 4707.

[22] C. L. Siegel and J. Moser, Lectures in Celestial Mechanics (Springer-Verlag, Berlin, 1971).

[23] N. Nekhoroshev, Russ. Math. Surv. 32, 1 (1977).

[24] A. Bazzani, S. Marmi, and G. Turchetti, Celest. Mech. 47, 333 (1990).

[25] G. Turchetti, in Proceedings of Number Theory and Physics, edited by J.M. Luck, P. Moussa, and M. Waldschmidt, Springer Proc. Phys. Vol. 47 (SpringerVerlag, Berlin, 1990), p. 223.

[26] J. S. Berg and F. Ruggiero, Report No. CERN SL-AP-9671, 1996.

[27] E. Métral and A. Verdier, Report No. CERN-AB-2004019-ABP, 2004.

[28] N. Cufaro Petroni, S. De Martino, S. De Siena, and F. Illuminati, Phys. Rev. E 72, 066502 (2005).

[29] C. K. Allen and T. P. Wangler, Phys. Rev. ST Accel. Beams 5, 124202 (2002).

[30] T. Sen, P. Lebrun, R. Moore, V. Shiltsev, M. Syphers, X. L. Zhang, W. Fischer, F. Schmidt, and F. Zimmermann, in Proceedings of 2003 Particle Accelerator Conference, edited by J. Chew, P. Lucas, and S. Webber (IEEE Service Center, Piscataway, NJ, 2003), p. 1754.

[31] V. Shiltsev, Y. Alexahin, V. Lebedev, P. Lebrun, R. S. Moore, T. Sen, A. Tollestrup, A. Valishev, and X. L. Zhang, Phys. Rev. ST Accel. Beams 8, 101001 (2005).

[32] E. Métral, at the LHC-CC10, 4th LHC Crab Cavity Workshop, CERN, 2010.

[33] F. Zimmermann, "SPS Emittance Growth in Coast" at the Machine Studies Working Group, 2010.

[34] E. Métral (private communication).

[35] A. Bazzani, G. Servizi, G. Turchetti, and E. Todesco, Report No. CERN-94-02, 1994. 